\title{
A New Quantum Behaved Particle Swarm Optimization
}

\author{
Millie Pant \\ Department of Paper Technology \\ IIT Roorkee, Saharanpur \\ India \\ millifpt@iitr.ernet.in
}

\author{
Radha Thangaraj \\ Department of Paper Technology \\ IIT Roorkee, Saharanpur \\ India \\ t.radha@ieee.org
}

\author{
Ajith Abraham \\ Q2S, Norwegian Center of Excellence \\ NTNU, Trondheim \\ Norway \\ ajith.abraham@ieee.org
}

\begin{abstract}
This paper presents a variant of Quantum behaved Particle Swarm Optimization (QPSO) named Q-QPSO for solving global optimization problems. The Q-QPSO algorithm is based on the characteristics of QPSO, and uses interpolation based recombination operator for generating a new solution vector in the search space. The performance of Q-QPSO is compared with Basic Particle Swarm Optimization (BPSO), QPSO and two other variants of QPSO taken from literature on six standard unconstrained, scalable benchmark problems. The experimental results show that the proposed algorithm outperforms the other algorithms quite significantly.
\end{abstract}

\section{Categories and Subject Descriptors}

D.3.3 [Programming Languages]: Language Contructs and Features - abstract data types, polymorphism

\section{General Terms}

Algorithms, Performance, Reliability, Experimentation

\section{Keywords}

Particle swarm optimization, Interpolation, Global optimization, Quantum behavior.

\section{INTRODUCTION}

Particle Swarm Optimization (PSO) is relatively a newer addition to a class of population based search technique for solving numerical optimization problems. The particles or members of the swarm fly through a multidimensional search space looking for a potential solution.

In classical (or original PSO), developed by Kennedy and Eberhart in 1995 [1], each particle adjusts its position in the search space from time to time according to the flying experience of its own and of its neighbors (or colleagues).

For a D-dimensional search space the position of the $i^{\text {th }}$ particle is represented as $\mathrm{X}_{\mathrm{i}}=\left(\mathrm{x}_{\mathrm{i} 1}, \mathrm{x}_{\mathrm{i} 2}, \ldots, \mathrm{x}_{\mathrm{iD}}\right)$. Each particle maintains a memory of its previous best position $P_{\text {besti }}=\left(p_{i 1}, p_{i 2} \ldots p_{i D}\right)$.

Permission to make digital or hard copies of all or part of this work for personal or classroom use is granted without fee provided that copies are not made or distributed for profit or commercial advantage and that copies bear this notice and the full citation on the first page. To copy otherwise, or republish, to post on servers or to redistribute to lists, requires prior specific permission and/or a fee.

GECCO'08, July 12--16, 2008, Atlanta, Georgia, USA.

Copyright 2008 ACM 978-1-60558-131-6/08/07...\$5.00.
The best one among all the particles in the population is represented as $\mathrm{P}_{\mathrm{gbest}}=\left(\mathrm{p}_{\mathrm{g} 1}, \mathrm{p}_{\mathrm{g} 2} \ldots \mathrm{p}_{\mathrm{gD}}\right)$. The velocity of each particle is represented as $\mathrm{V}_{\mathrm{i}}=\left(\mathrm{v}_{\mathrm{i} 1}, \mathrm{v}_{\mathrm{i} 2}, \ldots \mathrm{v}_{\mathrm{iD}}\right)$. In each iteration, the $\mathrm{P}$ vector of the particle with best fitness in the local neighborhood, designated $\mathrm{g}$, and the $\mathrm{P}$ vector of the current particle are combined to adjust the velocity along each dimension and a new position of the particle is determined using that velocity. The two basic equations which govern the working of PSO are that of velocity vector and position vector given by:

$$
\begin{aligned}
& v_{i d}=w v_{i d}+c_{1} r_{1}\left(p_{i d}-x_{i d}\right)+c_{2} r_{2}\left(p_{g d}-x_{i d}\right) \\
& x_{i d}=x_{i d}+v_{i d}
\end{aligned}
$$

The first part of equation (1) represents the inertia of the previous velocity, the second part is the cognition part and it tells us about the personal experience of the particle, the third part represents the cooperation among particles and is therefore named as the social component. Acceleration constants $c_{1}, c_{2}$ and inertia weight $\mathrm{w}$ are the predefined by the user and $\mathrm{r}_{1}, \mathrm{r}_{2}$ are the uniformly generated random numbers in the range of $[0,1]$.

PSO has undergone a plethora of changes since its development. One of the recent developments in PSO is the application of Quantum laws of mechanics to observe the behavior of PSO. Such PSO's are called Quantum PSO (QPSO). Some variants of QPSO include mutation based PSO [2], [3], where mutation is applied to the mbest (mean best) and gbest (global best) positions of the particle, also in one of the variants of QPSO a perturbation constant called Lyapunov constant is added. However to the best of our knowledge no has used the concept of recombination operator in QPSO.

This paper presents a QPSO called Q-QPSO which uses the quantum theory of mechanics to govern the movement of swarm particles along with an interpolation (quadratic interpolation) based recombination operator.

The concept of quadratic interpolation as a recombination operator was introduced by us [4], [5], for improving the performance of classical PSO, where it gave significantly good results. This motivated us to apply this concept for QPSO also to improve its performance.

The remaining of the paper is organized as follows: Section 2 briefly describes the Quantum Particle Swarm Optimization. Section 3, explains the proposed Q-QPSO, Section 4, gives the experimental settings and numerical results of some selected unconstrained benchmark problems. The paper finally concludes with Section 5. 


\section{QUANTUM PARTICLE SWARM OPTIMIZATION}

The development in the field of quantum mechanics is mainly due to the findings of Bohr, de Broglie, Schrödinger, Heisenberg and Bohn in the early twentieth century. Their studies forced the scientists to rethink the applicability of classical mechanics and the traditional understanding of the nature of motions of microscopic objects [6].

As per classical PSO, a particle is stated by its position vector $\mathrm{x}_{\mathrm{i}}$ and velocity vector $\mathrm{v}_{\mathrm{i}}$, which determine the trajectory of the particle. The particle moves along a determined trajectory following Newtonian mechanics. However if we consider quantum mechanics, then the term trajectory is meaningless, because $x_{i}$ and $v_{i}$ of a particle cannot be determined simultaneously according to uncertainty principle.

Therefore, if individual particles in a PSO system have quantum behavior, the performance of PSO will be far from that of classical PSO [7].

In the quantum model of a PSO, the state of a particle is depicted by wavefunction $\Psi(x, t)$, instead of position and velocity. The dynamic behavior of the particle is widely divergent from that of the particle in traditional PSO systems. In this context, the probability of the particle's appearing in position $x_{i}$ from probability density function $|\Psi(x, t)|^{2}$, the form of which depends on the potential field the particle lies in [2].

The particles move according to the following iterative equations [8], [9]:

$$
\begin{aligned}
& x(t+1)=p+\beta * \mid \text { mbest }-x(t) \mid * \ln (1 / u) \text { if } k \geq 0.5 \\
& x(t+1)=p-\beta * \mid \text { mbest }-x(t) \mid * \ln (1 / u) \text { if } k<0.5
\end{aligned}
$$

where

$$
\begin{aligned}
& p=\left(c_{1} p_{i d}+c_{2} P_{g d}\right) /\left(c_{1}+c_{2}\right) \\
& \text { mbest }=\frac{1}{M} \sum_{i-1}^{M} P_{i}=\left(\frac{1}{M} \sum_{i=1}^{M} P_{i 1}, \frac{1}{M} \sum_{i=1}^{M} P_{i 2}, \ldots . ., \frac{1}{M} \sum_{i=1}^{n} P_{i d}\right)
\end{aligned}
$$

Mean best (mbest) of the population is defined as the mean of the best positions of all particles, $\mathrm{u}, \mathrm{k}, \mathrm{c} 1$ and $\mathrm{c} 2$ are uniformly distributed random numbers in the interval $[0,1]$. The parameter $\beta$ is called contraction-expansion coefficient. The flow chart of QPSO algorithm is shown in Figure 1.

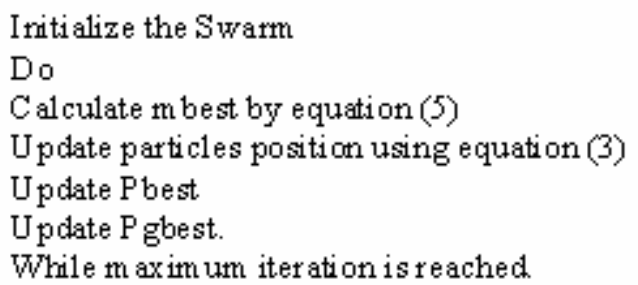

Figure 1 Flow of QPSO Algorithm

\section{PROPOSED Q-QPSO ALGORITHM}

The proposed Q-QPSO algorithm is a simple and modified version of QPSO in which we have introduced the concept of recombination. The Q-QPSO algorithm starts like the usual QPSO using equations (3), (4) and (5). At the end of each iteration, the quadratic interpolation recombination operator is invoked to generate a new swarm particle. The new particle is accepted in the swarm only if it is better than the worst particle (i.e. the particle having maximum fitness) present in the swarm. This process is repeated iteratively until a better solution is obtained.

The quadratic crossover operator is a nonlinear operator which produces a new solution vector lying at the point of minima of the quadratic curve passing through the three selected swarm particles.

The selection of particles, say $\{a, b, c\}$ is done as follows:

$\mathrm{a}=\mathrm{X}_{\min }$, (the swarm particle having minimum (or best) fitness function value)

$\{b, c\}=\{$ randomly chosen particles from the remaining members of the swarm.

( $a, b$ and $c$ should be three different particles)

The idea behind the inclusion of an interpolation operator is to facilitate the Q-QPSO with a recombination operator which will help in finding a new solution point in the search space. Since, we are always holding the particle having the best fitness function value to take part in recombination process, the probability of generating a new trial vector with fitness function which is better than at least one of the existing solution vectors in the swarm increases. Thus the new particle is accepted in the swarm only if it is better than the worst particle.

Mathematically, the new particle $\tilde{x}^{i}=\left(\tilde{x}^{1}, \tilde{x}^{2}, \ldots \ldots \tilde{x}^{n}\right)$, is given as:

$\tilde{x}^{i}=\frac{1}{2} \frac{\left(b^{i^{2}}-c^{i^{2}}\right) * f(a)+\left(c^{i^{2}}-a^{i^{2}}\right) * f(b)+\left(a^{i^{2}}-b^{i^{2}}\right) * f(c)}{\left(b^{i}-c^{i}\right) * f(a)+\left(c^{i}-a^{i}\right) * f(b)+\left(a^{i}-b^{i}\right) * f(c)}$

The computational steps of Q-QPSO algorithm are given by:

Step 1: Initialize the swarm with uniformly distributed random numbers.

Step 2: Calculate mbest using equation (5)

Step 3: Update particles position using equation (3)

Step 4: Evaluate the fitness value of each particle

Step 5: If the current fitness value is better than the best fitness value (Pbest) in history

Then Update Pbest by the current fitness value.

Step 6: Update Pgbest (global best)

Step 7: Find a new particle using equation (6)

Step 8: If the new particle is better than the worst particle in the swarm particle.

Then replace the worst particle by the new

Step 9: Go to step 2 until maximum iterations reached.

\section{EXPERIMENTAL SETTINGS AND BENCHMARK PROBLEMS}

In Q-QPSO algorithm a linearly decreasing contraction-expansion coefficient $(\beta)$ is used which starts at 1.0 and ends at 0.5 . the acceleration constants are taken as 2.0. In order to check the compatibility of the proposed Q-QPSO algorithm we have tested 
it on 10 benchmark problems (unconstrained) given in Table 1. All the test problems are highly multimodal and scalable in nature and are considered to be starting point for checking the credibility of any optimization algorithm.

There is not much literature available in which QPSO is used extensively for solving a variety of test problems. Therefore, for the present study, we have considered 10 test problems out of which the first three problems are the ones that have been tested with some variants of QPSO. The remaining 6 problems we have solved with our version and with QPSO and BPSO. As in [3], for functions $\mathrm{f} 1, \mathrm{f} 2$ and $\mathrm{f} 3$, three different dimension sizes are tested. They are 10, 20 and 30. The maximum number of generations is set as 1000, 1500 and 2000 corresponding to the dimensions 10, 20 and 30 respectively. Different population sizes are used for each function with different dimensions. The population sizes are
20, 40 and 80 . We have tested the functions $\mathrm{f} 4$ - f10 with dimensions 10, 30 and 50. A total of 30 runs for each experimental setting are conducted and the average fitness of the best solutions throughout the run is recorded.

Tables 2, 3 and 4 show the mean best fitness of Q-QPSO, BPSO, QPSO and its two variants in literature for functions $\mathrm{f} 1, \mathrm{f} 2$ and $\mathrm{f} 3$ respectively. Table 5 shows the mean best fitness values of QQPSO, BPSO and QPSO for the functions $\mathrm{f} 4$ - f10. Tables 6, 7 and 8 shows the T-test values for the benchmark problems $\mathrm{f} 1, \mathrm{f} 2$ and $\mathrm{f} 3$ in comparison with the other algorithms. Figures 2, 3 and 4 depict the performance with a focus on mean best fitness for some selected functions. In all the Tables, 'Pop' represents population, 'Dim' represents dimension and 'Gen' represents Generation.

Table 1. Numerical benchmark problems

\begin{tabular}{|l|c|c|}
\hline \multicolumn{1}{|c|}{ Function } & Range & Optimum \\
\hline$f_{1}(x)=\sum_{i=1}^{n}\left(x_{i}^{2}-10 \cos \left(2 \pi x_{i}\right)+10\right)$ & {$[2.56,5.12]$} & 0 \\
\hline$f_{2}(x)=\frac{1}{4000} \sum_{i=0}^{n-1} x_{i}^{2}-\prod_{i=0}^{n-1} \cos \left(\frac{x_{i}}{\sqrt{i+1}}\right)+1$ & {$[300,600]$} & 0 \\
\hline$f_{3}(x)=\sum_{i=0}^{n-1} 100\left(x_{i}+1-x_{i}^{2}\right)^{2}+\left(x_{i}-1\right)^{2}$ & {$[15,30]$} & 0 \\
\hline$f_{4}(x)=-\sum_{i=1}^{n} x_{i} \sin \left(\sqrt{\left|x_{i}\right|}\right)$ & {$[-500,500]$} & $-418.9829 * \mathrm{n}$ \\
\hline$f_{5}(x)=\left(\sum_{i=0}^{n-1}(i+1) x_{i}^{4}\right)+\operatorname{rand}[0,1]$ & {$[-1.28,1.28]$} & 0 \\
\hline$f_{6}(x)=20+e-20 \exp \left(-0.2 \sqrt{\frac{1}{n} \sum_{i=1}^{n} x_{i}^{2}}\right)-\exp \left(\frac{1}{n} \sum_{i=1}^{n} \cos \left(2 \pi x_{i}\right)\right)$ & {$[-32,32]$} & 0 \\
\hline$f_{7}(x)=\max \left|x_{i}\right|, \quad 0 \leq i<n$ & {$[-100,100]$} & 0 \\
\hline$f_{8}(x)=\sum_{i=0}^{n-1}\left\lfloor x_{i}+1 / 2\right]^{2}$ & {$[-100,100]$} & 0 \\
\hline$f_{9}(x)=\sum_{i=0}^{n-1}\left|x_{i}\right|+\prod_{i=0}^{n-1} x_{i}$ & {$[-10,10]$} & 0 \\
\hline$\sum_{i=0}^{n-1}\left(\sum_{j=0}^{i} x_{i}\right)^{2}$ & {$[-100,100]$} & \\
\hline
\end{tabular}


Table 2. Comparison results of function $\mathrm{f1}$ (Mean best)

\begin{tabular}{|c|c|c|c|c|c|c|c|c|c|}
\hline \multirow[t]{2}{*}{ Pop } & \multirow[t]{2}{*}{ Dim } & \multirow[t]{2}{*}{ Gen } & \multirow[t]{2}{*}{ Q-QPSO } & \multirow{2}{*}{$\begin{array}{c}\text { BPSO } \\
{[2]}\end{array}$} & \multirow{2}{*}{$\begin{array}{c}\text { QPSO } \\
{[2]}\end{array}$} & \multicolumn{2}{|c|}{$\begin{array}{c}\text { QPSO Mutation } \\
{[3]}\end{array}$} & \multicolumn{2}{|c|}{ QPSO Mutation [2] } \\
\hline & & & & & & Gbest & mbest & gbest & mbest \\
\hline \multirow{3}{*}{20} & 10 & 1000 & $4.915 \mathrm{e}-19$ & 5.5382 & 5.2543 & 5.2216 & 4.4788 & 4.3976 & 4.7332 \\
\hline & 20 & 1500 & 7.806e-19 & 23.1544 & 16.2673 & 16.1562 & 15.6535 & 14.1678 & 13.6202 \\
\hline & 30 & 2000 & 6.071e-19 & 47.4168 & 31.4576 & 26.2507 & 27.8098 & 25.6415 & 27.7975 \\
\hline \multirow{3}{*}{40} & 10 & 1000 & $6.550 \mathrm{e}-13$ & 3.5778 & 3.5685 & 3.3361 & 3.3837 & 3.2046 & 2.8160 \\
\hline & 20 & 1500 & $8.673 e-19$ & 16.4337 & 11.1351 & 10.9072 & 11.0128 & 9.5793 & 9.9143 \\
\hline & 30 & 2000 & $5.493 \mathrm{e}-19$ & 37.2896 & 22.9594 & 19.6360 & 21.0179 & 20.5479 & 19.8991 \\
\hline \multirow{3}{*}{80} & 10 & 1000 & 0.86794 & 2.5646 & 2.1245 & 2.0185 & 2.1833 & 1.7166 & 1.8923 \\
\hline & 20 & 1500 & 0.97712 & 13.3826 & 10.2759 & 7.7928 & 8.0755 & 7.2041 & 7.8625 \\
\hline & 30 & 2000 & $5.493 \mathrm{e}-19$ & 28.6293 & 16.7768 & 14.9055 & 14.9965 & 15.0393 & 15.4082 \\
\hline
\end{tabular}

Table 3. Comparison results of function $\mathrm{f} 2$ (Mean best)

\begin{tabular}{|c|c|c|c|c|c|c|c|c|c|}
\hline \multirow{2}{*}{ Pop } & \multirow{2}{*}{ Dim } & \multirow{2}{*}{ Gen } & \multirow{2}{*}{ Q-QPSO } & \multirow{2}{*}{ BPSO[2] } & \multirow{2}{*}{ QPSO[2] } & \multicolumn{2}{|c|}{ QPSO Mutation [3] } & \multicolumn{2}{|c|}{ QPSO Mutation[2] } \\
\cline { 6 - 10 } & & & & & & gbest & mbest & gbest & mbest \\
\hline \multirow{3}{*}{20} & 10 & 1000 & $\mathbf{0 . 0 6 2 6 5 7}$ & 0.09217 & 0.08331 & 0.0627 & 0.0732 & 0.0780 & 0.0932 \\
\cline { 2 - 9 } & 20 & 1500 & $\mathbf{0 . 0 0 5 0 9 1}$ & 0.03002 & 0.02033 & 0.0209 & 0.0189 & 0.0235 & 0.0193 \\
\cline { 2 - 9 } & 30 & 2000 & 0.015442 & 0.01811 & 0.01119 & 0.0110 & 0.0103 & $\mathbf{0 . 0 0 9 9}$ & 0.0114 \\
\hline \multirow{3}{*}{40} & 10 & 1000 & 0.057393 & 0.08496 & 0.06912 & 0.0539 & $\mathbf{0 . 0 5 2 0}$ & 0.0641 & 0.0560 \\
\cline { 2 - 9 } & 20 & 1500 & $\mathbf{0 . 0 0 5 8 2 7}$ & 0.02719 & 0.01666 & 0.0238 & 0.0247 & 0.0191 & 0.0171 \\
\cline { 2 - 9 } & 30 & 2000 & $\mathbf{0 . 0 0 7 8 7 4}$ & 0.01267 & 0.01161 & 0.0119 & 0.0105 & 0.0098 & 0.0092 \\
\hline \multirow{3}{*}{80} & 10 & 1000 & $\mathbf{0 . 0 3 1 5 2 7}$ & 0.07484 & 0.03508 & 0.0419 & 0.0542 & 0.0460 & 0.0554 \\
\cline { 2 - 9 } & 20 & 1500 & $\mathbf{0 . 0 0 6 6 3 3}$ & 0.02854 & 0.01460 & 0.0136 & 0.0194 & 0.0186 & 0.0123 \\
\cline { 2 - 9 } & 30 & 2000 & $\mathbf{0 . 0 0 6 6 4 8}$ & 0.01258 & 0.01136 & 0.0120 & 0.0082 & 0.0069 & 0.0111 \\
\hline
\end{tabular}

Table 4. Comparison results of function $\mathrm{f} 3$ (Mean best)

\begin{tabular}{|c|c|c|c|c|c|c|c|c|c|}
\hline \multirow{2}{*}{ Pop } & \multirow{2}{*}{ Dim } & \multirow{2}{*}{ Gen } & \multirow{2}{*}{ Q-QPSO } & \multirow{2}{*}{$\begin{array}{c}\text { BPSO } \\
{[2]}\end{array}$} & \multirow{2}{*}{$\begin{array}{c}\text { QPSO } \\
\text { [2] }\end{array}$} & \multicolumn{2}{|c|}{ QPSO Mutation [3] } & \multicolumn{2}{|c|}{ QPSO Mutation [2] } \\
\hline & & & & & & Gbest & mbest & gbest & mbest \\
\hline \multirow{3}{*}{20} & 10 & 1000 & 5.544203 & 94.1276 & 59.4764 & 27.4620 & 22.1870 & 21.2081 & 15.3939 \\
\hline & 20 & 1500 & 15.538104 & 204.336 & 110.664 & 49.1176 & 68.4096 & 61.9268 & 67.6978 \\
\hline & 30 & 2000 & 25.687072 & 313.734 & 147.609 & 97.5952 & 113.3080 & 86.1195 & 76.1894 \\
\hline \multirow{3}{*}{40} & 10 & 1000 & 4.200496 & 71.0239 & 10.4238 & 7.8741 & 7.9850 & 8.1828 & 9.5005 \\
\hline & 20 & 1500 & 14.158022 & 179.291 & 46.5957 & 28.4435 & 52.9333 & 40.0749 & 55.4853 \\
\hline & 30 & 2000 & 24.126324 & 289.593 & 59.0291 & 62.3854 & 64.1942 & 65.2891 & 68.0551 \\
\hline \multirow{3}{*}{80} & 10 & 1000 & 2.893087 & 37.3747 & 8.63638 & 6.7098 & 5.7159 & 7.3686 & 6.4841 \\
\hline & 20 & 1500 & 12.033052 & 83.6931 & 35.8947 & 31.0929 & 24.4566 & 30.1607 & 38.3067 \\
\hline & 30 & 2000 & 22.426013 & 202.672 & 51.5479 & 43.7622 & 45.2270 & 38.3036 & 52.4678 \\
\hline
\end{tabular}


Table 5. Comparison results of functions $\mathrm{f} 4 \mathrm{-}-\mathrm{f10}$ (Mean best)

\begin{tabular}{|c|c|c|c|c|c|}
\hline Function & Dim & Gen & BPSO & QPSO & Q-QPSO \\
\hline \multirow{3}{*}{$\mathrm{f} 4$} & 10 & 1000 & -2389.365 & -3871.03 & -3898.67 \\
\hline & 30 & 2000 & -6466.188 & -8967.29 & -9998 \\
\hline & 50 & 3000 & -10473.09 & -13105.9 & -14783.6 \\
\hline \multirow{3}{*}{ f5 } & 10 & 1000 & 0.502671 & 0.452975 & 0.376021 \\
\hline & 30 & 2000 & 0.617222 & 0.501799 & 0.497801 \\
\hline & 50 & 3000 & 0.788322 & 0.598823 & 0.537782 \\
\hline \multirow{3}{*}{ f6 } & 10 & 1000 & $6.965 \mathrm{e}-12$ & $4.407 e-015$ & $1.210 \mathrm{e}-015$ \\
\hline & 30 & 2000 & $3.618 \mathrm{e}-05$ & $7.568 \mathrm{e}-012$ & $5.828 \mathrm{e}-015$ \\
\hline & 50 & 3000 & 3.43866 & $1.018 \mathrm{e}-007$ & $6.267 \mathrm{e}-014$ \\
\hline \multirow{3}{*}{$\mathrm{f} 7$} & 10 & 1000 & $3.79373 \mathrm{e}-006$ & $1.09816 \mathrm{e}-09$ & $7.93766 \mathrm{e}-013$ \\
\hline & 30 & 2000 & 7.836 & 4.11969 & 0.00571485 \\
\hline & 50 & 3000 & 27.8394 & 21.1619 & 0.2213 \\
\hline \multirow{3}{*}{ f8 } & 10 & 1000 & 0.00000 & 0.00000 & 0.00000 \\
\hline & 30 & 2000 & 0.05 & 0.00000 & 0.00000 \\
\hline & 50 & 3000 & 1.7 & 0.1 & 0.00000 \\
\hline \multirow{3}{*}{ f9 } & 10 & 1000 & $2.4148 \mathrm{e}-14$ & $2.02058 \mathrm{e}-32$ & 3.32734e-34 \\
\hline & 30 & 2000 & $2.04745 \mathrm{e}-07$ & $3.2466 \mathrm{e}-13$ & 7.94348e-16 \\
\hline & 50 & 3000 & 7.73949 & $1.67575 \mathrm{e}-08$ & 8.66751e-11 \\
\hline \multirow{3}{*}{$\mathrm{f} 10$} & 10 & 1000 & $2.51528 \mathrm{e}-22$ & $5.51618 \mathrm{e}-54$ & $1.00925 \mathrm{e}-62$ \\
\hline & 30 & 2000 & $1.34381 \mathrm{e}-07$ & $1.44342 \mathrm{e}-21$ & $2.9990 \mathrm{e}-27$ \\
\hline & 50 & 3000 & 0.00176764 & $1.06795 \mathrm{e}-10$ & $3.78622 \mathrm{e}-17$ \\
\hline
\end{tabular}

Table 6. T-test* value for the function $\mathrm{f1}$ : comparison of Q-QPSO with other algorithms

\begin{tabular}{|c|c|c|c|c|c|c|c|c|}
\hline \multirow{2}{*}{ Pop } & \multirow{2}{*}{ Dim } & \multirow{2}{*}{ Gen } & \multirow{2}{*}{$\begin{array}{c}\text { BPSO } \\
{[2]}\end{array}$} & \multirow{2}{*}{$\begin{array}{c}\text { QPSO } \\
{[2]}\end{array}$} & \multicolumn{2}{|c|}{ QPSO Mutation [3] } & \multicolumn{2}{|c|}{ QPSO Mutation [2] } \\
\hline & & & & & gbest & mbest & gbest & mbest \\
\hline \multirow{3}{*}{20} & 10 & 1000 & 9.9530 & 9.9402 & 13.5224 & 10.3788 & 9.5110 & 9.8434 \\
\hline & 20 & 1500 & 12.1083 & 14.9068 & 10.8334 & 8.9853 & 15.7493 & 13.3406 \\
\hline & 30 & 2000 & 15.1352 & 22.4110 & 16.9158 & 7.6914 & 21.0956 & 20.911 \\
\hline \multirow{3}{*}{40} & 10 & 1000 & 9.1640 & 9.4523 & 7.7164 & 6.8459 & 5.7384 & 8.1806 \\
\hline & 20 & 1500 & 16.4220 & 16.9198 & 11.2975 & 12.5773 & 18.6672 & 16.8778 \\
\hline & 30 & 2000 & 14.2989 & 17.3561 & 25.1958 & 7.4558 & 22.4234 & 24.0754 \\
\hline \multirow{3}{*}{80} & 10 & 1000 & 2.5229 & 1.9482 & 1.69199 & 1.8635 & 1.2992 & 1.5181 \\
\hline & 20 & 1500 & 7.2362 & 6.5989 & 6.6325 & 9.2566 & 7.2967 & 7.7037 \\
\hline & 30 & 2000 & 15.1607 & 20.4847 & 14.3885 & 19.2611 & 19.7066 & 18.6037 \\
\hline
\end{tabular}


Table 7. T-test* value for the function f2: comparison of Q-QPSO with other algorithms

\begin{tabular}{|c|c|c|c|c|c|c|c|c|}
\hline \multirow{2}{*}{ Pop } & \multirow{2}{*}{ Dim } & \multirow{2}{*}{ Gen } & \multirow{2}{*}{$\begin{array}{c}\text { BPSO } \\
{[2]}\end{array}$} & \multirow{2}{*}{$\begin{array}{c}\text { QPSO } \\
\text { [2] }\end{array}$} & \multicolumn{2}{|c|}{$\begin{array}{c}\text { QPSO } \\
\text { Mutation[3] }\end{array}$} & \multicolumn{2}{|c|}{$\begin{array}{c}\text { QPSO Mutation } \\
{[2]}\end{array}$} \\
\hline & & & & & gbest & mbest & gbest & mbest \\
\hline \multirow{3}{*}{20} & 10 & 1000 & 0.6204 & 0.4417 & 0.00093 & 0.2277 & 0.3304 & 0.6398 \\
\hline & 20 & 1500 & 4.2078 & 3.6522 & 4.2408 & 4.2339 & 4.6468 & 5.1432 \\
\hline & 30 & 2000 & 0.2525 & -0.4289 & -0.4429 & -0.523 & -0.568 & -0.4077 \\
\hline \multirow{3}{*}{40} & 10 & 1000 & 0.8184 & 0.3627 & -0.1081 & -0.1695 & 0.2101 & -0.0435 \\
\hline & 20 & 1500 & 4.5453 & 3.2313 & 4.8420 & 2.2757 & 4.2572 & 3.3365 \\
\hline & 30 & 2000 & 1.6119 & 1.4395 & 1.2345 & 1.0282 & 0.6970 & 0.5324 \\
\hline \multirow{3}{*}{80} & 10 & 1000 & 1.9956 & 0.1995 & 0.5749 & 1.1770 & 0.8125 & 1.2151 \\
\hline & 20 & 1500 & 4.2989 & 2.9130 & 2.2932 & 3.2294 & 3.6094 & 2.0360 \\
\hline & 30 & 2000 & 2.1889 & 2.0719 & 1.7473 & 0.5976 & 0.1293 & 1.7143 \\
\hline
\end{tabular}

Table 8. T-test* value for the function f3: comparison of Q-QPSO with other algorithms

\begin{tabular}{|c|c|c|c|c|c|c|c|c|}
\hline \multirow{2}{*}{ Pop } & \multirow{2}{*}{ Dim } & Gen & \multirow{2}{*}{ BPSO [2] } & \multirow{2}{*}{ QPSO [2] } & \multicolumn{2}{|c|}{ QPSO Mutation [3] } & \multicolumn{2}{|c|}{ QPSO Mutation [2] } \\
\cline { 4 - 9 } & & & & & gbest & mbest & gbest & mbest \\
\cline { 2 - 9 } & 20 & 1000 & 2.4962 & 1.9296 & 2.3366 & 1.6658 & 1.4285 & 1.5366 \\
\cline { 2 - 9 } & 30 & 2000 & 2.8828 & 3.1750 & 2.7207 & 3.1501 & 2.5931 & 2.3971 \\
\hline \multirow{3}{*}{40} & 10 & 1000 & 2.1021 & 2.3534 & 1.9774 & 2.3377 & 2.6069 & 3.0377 \\
\cline { 2 - 9 } & 20 & 1500 & 2.3963 & 4.4938 & 2.6616 & 3.4119 & 2.0751 & 3.5739 \\
\cline { 2 - 9 } & 30 & 2000 & 3.0378 & 3.0108 & 3.8951 & 4.1699 & 2.8380 & 4.4003 \\
\hline \multirow{2}{*}{80} & 10 & 1000 & 3.2847 & 1.8772 & 2.5347 & 2.4382 & 2.8315 & 3.2502 \\
\cline { 2 - 9 } & 20 & 1500 & 2.8594 & 3.5828 & 3.2790 & 2.5388 & 2.9890 & 3.7068 \\
\hline
\end{tabular}

* The $\mathrm{t}$ value of 29 degrees of freedom is significant at a level a 0.05 level of significance by a two-tailed $t$-test 


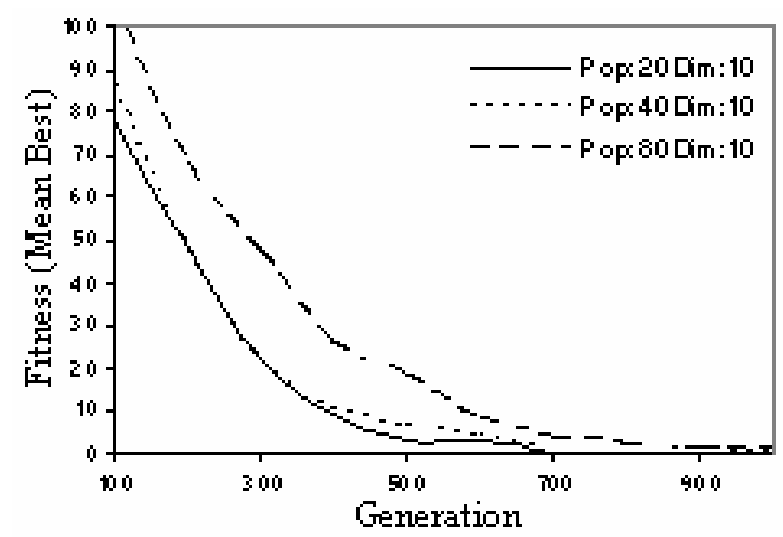

(a)

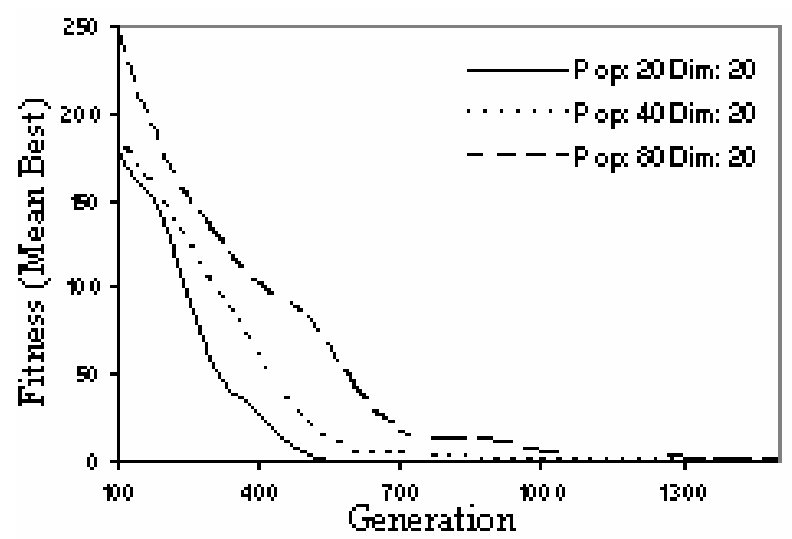

(b)

Figure 2. Performance for Rastringin function (a) dimension 10 (b) dimension 20

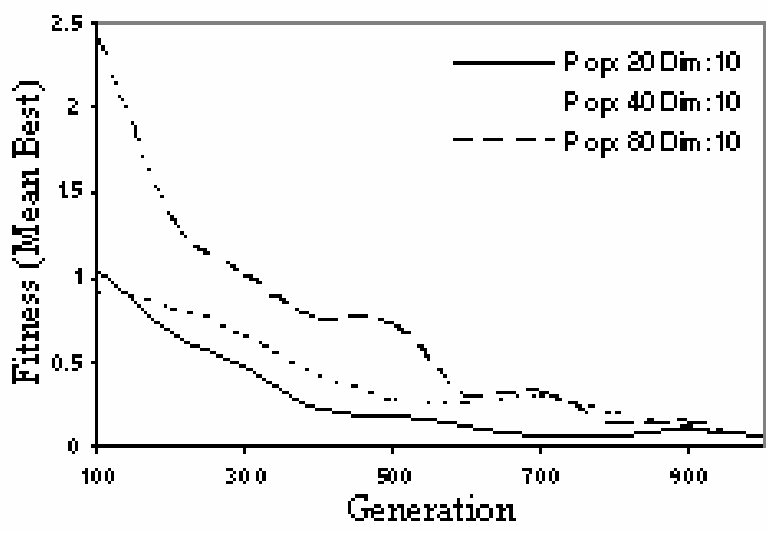

(a)

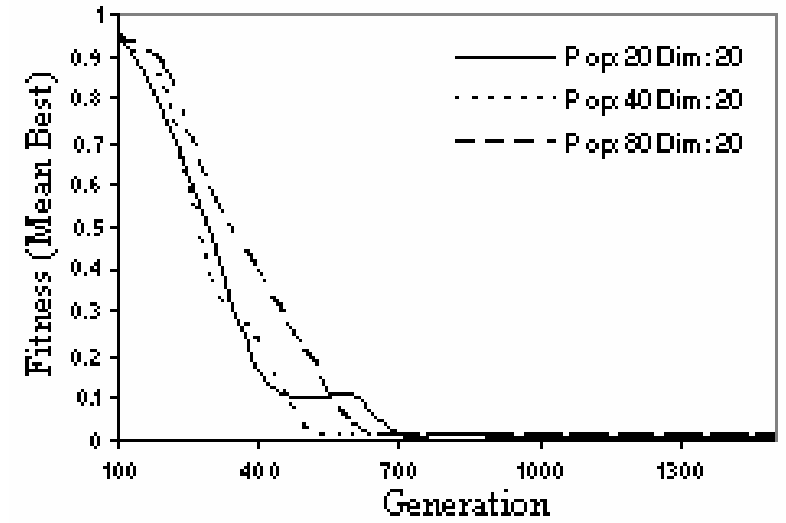

(b)

Figure 3. Performance for Griewank function (a) dimension 10 (b) dimension 20

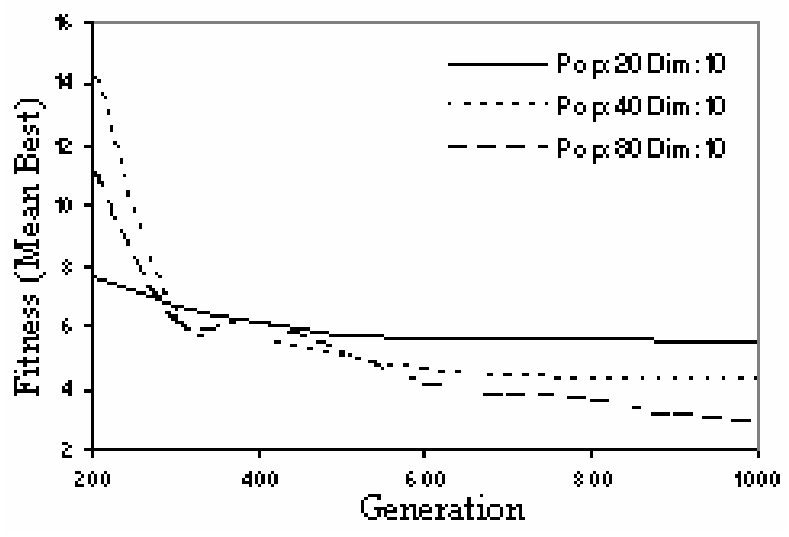

(a)

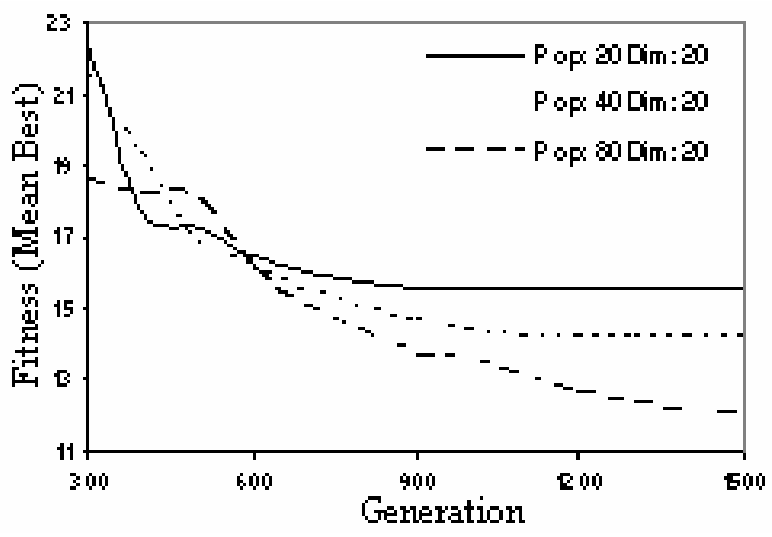

(b)

Figure 4. Performance for Rosenbrock function (a) dimension 10 (b) dimension 20 


\section{CONCLUSIONS}

This paper presents a variant of Quantum PSO called Q-QPSO, incorporating the concept of recombination operator. The proposed Q-QPSO is tested on three standard unconstrained benchmark test problems and the results are compared with some of the existing QPSO (containing mutation operator) and standard PSO.

For the first test problem, which is, Rastringin's function (a highly multimodal function), the proposed Q-QPSO algorithm outperformed all the given algorithms, quite significantly and gave the near optimum solution (which is 0 ) in all the test cases. Similarly for the second test problem (Griewank function), QQPSO gave better results than the other algorithms in seven out of the nine test cases tried. For the test functions f3 - f10 also QQPSO gave superior results in all the cases. The dominance of the proposed Q-QPSO algorithm is also apparent from the two tailed t-test given in Tables 5, 6 and 7 .

However, we would like to add that the collection of test problems taken in this paper is not exhaustive and therefore making any concrete conclusion on the performance of Q-QPSO do not sound justified. Moreover, it is very much possible that the inclusion of a crossover operator may have an adverse effect on the diversity of the algorithm which in turn may deteriorate its performance for solving problems having large number of variables. Also, it would be interesting to do a theoretical analysis on quadratic interpolation operator and its application to optimization problems.

However, on the positive side it is quite evident from the numerical results that for the highly multimodal problems having variables up to 50, Q-QPSO is definitely a better choice over the contemporary optimization algorithms.

For the future work, we shall be apply Q-QPSO for solving more complex unconstrained and constrained optimization problems. Also we shall be studying the combined effect of mutation and recombination on a Quantum behaved PSO.

\section{REFERENCES}

[1] Kennedy, J. and Eberhart, R. Particle Swarm Optimization. IEEE International Conference on Neural Networks (Perth, Australia), IEEE Service Center, Piscataway, NJ, IV: 19421948, 1995.

[2] Liu J, Sun J, Xu W, Quantum-Behaved Particle Swarm Optimization with Adaptive Mutation Operator. ICNC 2006, Part I, Springer-Verlag: 959 - 967, 2006.

[3] Liu J, Xu W, Sun J. Quantum-Behaved Particle Swarm Optimization with Mutation Operator. In Proc. of the $17^{\text {th }}$ IEEE Int. Conf. on Tools with Artificial Intelligence, Hong Kong (China), 2005.

[4] Millie Pant, Radha Thangaraj and Ajith Abraham, A New PSO Algorithm with Crossover Operator for Global Optimization Problems, Second International Symposium on Hybrid Artificial Intelligent Systems (HAIS'07), Advances in Softcomputing Series, Springer Verlag, Germany, E. Corchado et al. (Eds.): Innovations in Hybrid Intelligent Systems, Vol. 44, pp. 215-222, 2007.

[5] Millie Pant, Radha Thangaraj and Ajith Abraham, A New Particle Swarm Optimization Algorithm Incorporating
Reproduction Operator for Solving Global Optimization Problems, 7th International Conference on Hybrid Intelligent Systems, Kaiserslautern, Germany, IEEE Computer Society press, USA, ISBN 07695-2662-4, pp. 144-149, 2007.

[6] Pang XF, Quantum mechanics in nonlinear systems. River Edge (NJ, USA): World Scientific Publishing Company, 2005.

[7] Bin Feng, Wenbo Xu, Adaptive Particle Swarm Optimization Based on Quantum Oscillator Model. In Proc. of the 2004 IEEE Conf. on Cybernetics and Intelligent Systems, Singapore: 291 - 294, 2004.

[8] Sun J, Feng B, Xu W, Particle Swarm Optimization with particles having Quantum Behavior. In Proc. of Congress on Evolutionary Computation, Portland (OR, USA), 325 - 331, 2004.

[9] Sun J, Xu W, Feng B, A Global Search Strategy of QuantumBehaved Particle Swarm Optimization. In Proc. of the 2004 IEEE Conf. on Cybernetics and Intelligent Systems, Singapore: 291 - 294, 2004 